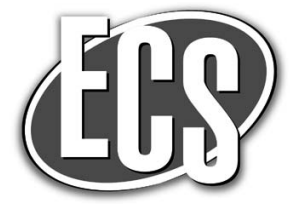

\title{
Near-Shore Aggregation Mechanism of Electrolyte Decomposition Products to Explain Solid Electrolyte Interphase Formation
}

\author{
Keisuke Ushirogata, ${ }^{\text {a,b }}$ Keitaro Sodeyama, ${ }^{\text {b,c }}$ Zdenek Futera, ${ }^{\text {b }}$ Yoshitaka Tateyama,,${ }^{\text {, c,d,z }}$ \\ and Yukihiro Okuno ${ }^{\mathrm{a}, \mathrm{b}, \mathrm{z}}$ \\ ${ }^{a}$ Research and Development Management Headquarters, FUJIFILM Corporation, Minamiashigara, \\ Kanagawa 250-0193, Japan \\ ${ }^{b}$ International Center for Materials Nanoarchitectonics (MANA), National Institute for Materials Science (NIMS), \\ Tsukuba, Ibaraki 305-0044, Japan \\ ${ }^{c}$ Elements Strategy Initiative for Catalysts \& Batteries, Kyoto University, Nishikyo-ku, Kyoto 615-8245, Japan \\ ${ }^{d}$ PRESTO, Japan Science and Technology Agency (JST), 4-1-8 Honcho, Kawaguchi, Saitama 333-0012, Japan
}

\begin{abstract}
To get insight of the formation mechanism of solid electrolyte interphase (SEI) film in Lithium-ion battery (LIB), we examine a probable scenario, referred to as "surface growth mechanism," for electrolyte involving ethylene carbonate (EC) solvent and vinylene carbonate (VC) additive by using density functional theory (DFT). We first extracted stable SEI film components (SFCs) for the EC/VC electrolyte and constructed probable SFC aggregates via DFT molecular dynamics. We then examined their solubility in the EC solution, their adhesion to a model graphite electrode, and the electronic properties. The results showed that the SFC aggregates are characterized by "unstable adhesion" to the graphite surface and "high electronic insulation" against the EC solution. These characteristics preclude explaining SEI growth up to a typical thickness of several tens of nanometers based on the surface growth mechanism. With the present results, we propose "near-shore aggregation" mechanism, where the SFCs formed at the electrode surface desorb into the near-shore region and form aggregates. The SFC aggregates coalesce and come into contact with the electrode to complete the SEI formation. The present model provides a novel perspective for the long-standing problem of SEI formation. (C) The Author(s) 2015. Published by ECS. This is an open access article distributed under the terms of the Creative Commons Attribution 4.0 License (CC BY, http://creativecommons.org/licenses/by/4.0/), which permits unrestricted reuse of the work in any medium, provided the original work is properly cited. [DOI: 10.1149/2.0301514jes] All rights reserved.
\end{abstract}

Manuscript submitted June 18, 2015; revised manuscript received September 3, 2015. Published October 7, 2015.

Lithium-ion batteries (LIBs) have attracted considerable attention for use in larger power sources like electric vehicles and energy storage system because of their high energy densities. ${ }^{1,2}$ For such use, a higher degree of safety, a longer cycle life, higher voltage and capacity will be indispensable in the future. An important key of the stability and durability of the LIB is the solid electrolyte interphase (SEI) formed at the negative electrode-electrolyte interface. ${ }^{3,4}$ It is generally accepted that molecules in the electrolyte solution reductively decompose to form various SEI film components (SFCs), such as organic oligomers (e.g., $\left(\mathrm{CH}_{2} \mathrm{OCO}_{2} \mathrm{Li}\right)_{2}$, and $\left.\mathrm{ROCO}_{2} \mathrm{Li}\right)$ and inorganic moieties (e.g., $\left.\mathrm{Li}_{2} \mathrm{CO}_{3}, \mathrm{LiF}\right)$ at the first charging, ${ }^{5}$ and that the SFCs precipitate on the electrode surface to form a stable SEI film with a thickness of several tens of nanometers. ${ }^{6}$

The SEI hinders electron transport from the electrode to the electrolyte solution, preventing further electrolyte decomposition, while allowing $\mathrm{Li}^{+}$ion transport. This property decreases the irreversible capacity and improves the safety of LIBs. Additives to the electrolyte solution also have a large impact on SEI performance. Even a small amount of additive up to a few wt $\%$ significantly improves the irreversible capacity and cyclability. ${ }^{7}$ In general, the additive molecules modify the SFCs and lead to different properties of the SEI film. Despite the important roles of the SEI in LIB operation, the microscopic formation processes are still unclear because of the difficulty in operando observations of chemical reactions at the electrodeelectrolyte interfaces.

The detailed formation processes of SEI have been typically assumed to involve "surface growth mechanism", mainly in the first cycle. The first atomistic step is reductive decomposition of the electrolyte molecules, followed by formation of fundamental SFCs with the decomposed products. Precipitation of the SFCs on the electrode surface is the next step. At this stage, the bonds between the SFCs and the electrode surface as well as among the SFCs play crucial roles. At a certain thickness of the SFC aggregate, the electronic insulation begins to suppress electron flow from the electrode to the electrolyte solution and further reductive decomposition of the electrolyte molecules. Growth of the SFC aggregates then stops, and the formation of the SEI film in the first cycle, which is most dominant, is completed.
However, there is still a question as to how the reductive decompositions of the electrolyte are repeated until the thickness reaches several tens of nanometers. ${ }^{6}$ The surface growth mechanism implies that the electronic insulation of SEI made by ethylene carbonate (EC) solvents only is not good enough to prevent electron tunneling. The effect of vinylene carbonate (VC) additives, which usually gives thinner SEI thickness ${ }^{8,9}$ can be also explained by better blocking of electron tunneling in the VC-derived SFC aggregates and thus in the SEI. Although this explanation sounds very reasonable, the mechanism has not yet been identified.

Many studies have addressed the formation of SEI and its properties. ${ }^{3-40}$ In the theoretical and computational researches, however, most focused on the first step of the reductive decompositions and the subsequent processes have been less explored. Recently, some theoretical studies on the solubility and adhesion properties of SFCs have been conducted by use of classical molecular dynamics (MD) ${ }^{24-28}$ or density functional theory (DFT) calculations with cluster boundary condition (CBC). ${ }^{31}$ With classical MD, however, it is difficult to treat the modulation of electronic charge where the solvation environment is changing. Previous CBC-DFT studies on the stability of adhesion to graphite surfaces did not include the environmental solvent, consideration of which is essential because solvation by the electrolyte molecules always competes with adhesion. Furthermore, temperature effects also influence adhesion properties. It is therefore desirable to treat these quantities with DFT-MD simulations that explicitly include electrolyte molecules at the working temperature of the battery.

In this study, we investigated the whole processes denoted above, from SFC generation to the formation of SEI seeds, by DFT calculations. Adopting typical electrolyte solutions with EC solvent and VC additive, we examined the reductive decomposition and subsequent oligomerization of the electrolyte molecules, and extracted minimum models of the probable SFCs. At this stage we considered possible binding among EC- and VC-derived radicals as well as the intact $\mathrm{EC}$ and $\mathrm{VC}$ molecules. For example, dilithium ethylene dicarbonate, $\left(\mathrm{Li}_{2} \mathrm{EDC}\right)$, is a candidate in the EC-only case. ${ }^{5}$ Here we extensively explored the SFCs associated with VC additives by the CBC-DFT calculations in conjunction with our previous free energy analysis. ${ }^{35}$

We then used DFT-MD samplings to construct the probable SFC aggregates, taking dynamical and morphological characteristics into account. We also examined the solubility properties into the EC 
solution, a measure of aggregation tendency, and the adhesion to the edges of possible reduced graphite. The electronic insulating properties of the SFC aggregates on the electrode were also clarified. On the basis of all the results, we discuss how SEI films grow and when the growth stops in the present modeling of the organic SFCs. Finally, we propose a new probable atomistic mechanism for the formation of the SEI films.

\section{Calculation}

DFT molecular dynamics. - We used DFT-MD samplings to evaluate the average characteristics of the aggregation, adhesion and electronic states of SFCs. In this work, we chose EC solvent and VC additive (Figure 1) for the electrolyte components because they are representative and the products of their reductive decompositions are likely to be major organic SFCs. The other electrolyte components such as dimethyl carbonate (DMC) and diethyl carbonate (DEC) that do not decompose are neglected for simplicity. Since more dielectric molecules such as EC and VC are expected to preferentially exist near the charged electrode surface, the present assumption and modeling is a reasonable first step for the SEI formation mechanisms.

Structural stabilities of the SFC monomers and aggregates were estimated via DFT-MD energy samplings with the periodic boundary condition (PBC). Aggregation tendency was evaluated on the basis of the dissolution energy of the SFC in the EC solution. We examined monomer adsorption and aggregate adhesion via supercells involving the edge surfaces of the graphite electrode as well as EC solvent molecules. The projected densities of states (PDOSs) of these supercells were also used to determine the electronic insulating properties of the SFCs in the EC solution.

The computational details of the DFT-MD are as follows. The $\mathrm{PBC}$ was adopted to deal with the liquid state at constant density. We used Car-Parrinello type of DFT-MD simulations, ${ }^{41}$ with CPMD code. $^{42}$ A fictitious electronic mass of 600 a.u. and a time step of 5 a.u. (0.12 fs) were chosen. The system temperature was controlled using a Nosé thermostat ${ }^{43,44}$ with a target temperature of $353 \mathrm{~K}$. After equilibration, statistical averages were computed from trajectories of at least $5 \mathrm{ps}$ in length. The electronic wave function was quenched to the Born-Oppenheimer surface about every 1 ps to maintain adiabaticity. We used the PBE exchange-correlation functional. ${ }^{45,46}$ The energy cutoff of the plane wave basis set was set to $90 \mathrm{Ry}$. We used Stefan Goedecker's norm-conserving pseudopotentials for $\mathrm{C}, \mathrm{H}, \mathrm{O}$ and $\mathrm{Li}^{4}{ }^{4-49}$ Calculations with DFT-D scheme that involves van der Waals $(\mathrm{vdW})$ interaction were used in a part of the calculations. ${ }^{50}$

Aggregation and dissolution energy. - To estimate the aggregation/dissolution preferences of SFCs in the EC solution, we calculated the dissolution energy, $E_{\mathrm{diss}}$, approximately using the following formula,

$$
E_{\mathrm{diss}}=E(m \mathrm{EC}+\mathrm{SFC})-m \mu_{\mathrm{EC}}-\mu_{\mathrm{SFC}}
$$

where $\mathrm{E}(m \mathrm{EC}+\mathrm{SFC})$ is the equilibrium total energy of the target system with $m \mathrm{EC}$ solvent molecules and one SFC, and $\mu \mathrm{EC}$ and $\mu \mathrm{SFC}$ correspond to the average chemical potentials of an $\mathrm{EC}$ molecule in the equilibrium EC solution and a SFC monomer in the SFC condensed phase, respectively, in the DFT-MD simulations. Positive (negative) dissolution energy indicates that aggregation of the SFCs is energetically favorable (unfavorable).

The chemical potential $\mu_{\mathrm{EC}}$ was calculated with a cubic supercell involving $64 \mathrm{EC}$ solvent molecules in which we adopted a cell dimension of $19.2109 \AA$ based on the density of $1.32 \mathrm{~g} / \mathrm{cm}^{3} .{ }^{51,52} \mathrm{On}$ the other hand, $\mu_{\mathrm{SFC}}$ was calculated by using another cubic supercell involving 40 or 60 model SFCs, depending on the SFC size. The sizes of supercells for the SFC condensed phase were determined based on the reported density of $\mathrm{Li}_{2} \mathrm{EDC}$, the most typical EC-derived SFC, of $1.86 \mathrm{~g} / \mathrm{cm}^{3},{ }^{24}$ which was obtained with classical MD calculations under ambient conditions. Although the equilibrium density may vary for the other SFC species, we expect that the small deviations of the calculated properties did not compromise our calculations. For the a<smiles>O=C1CCCO1</smiles>

EC b<smiles></smiles>

VC

Figure 1. Structures of (a) ethylene carbonate (EC) and (b) vinylene carbonate (VC) with the labels used throughout this paper.

average total energy $\mathrm{E}(m \mathrm{EC}+\mathrm{SFC})$, we used a cubic supercell with $64 \mathrm{EC}$ solvents at equilibrium and replaced $l(=64-m)$ EC solvent molecules with one SFC for the initial configuration, where $l$ corresponds to the number of EC and VC moieties in the inserted SFC. To prepare appropriate initial configurations, we carried out classical MD simulations for $3 \mathrm{~ns}$ and randomly extracted five condensed configurations, which have amorphous structures.

Adhesive energy. - We calculated the adhesive energies of the SFC monomers and aggregates at the interface between the graphite electrode and EC solvent by DFT-MD sampling. The adhesive energy was defined as the difference of the averaged total energy between the attached state on the surface and the dissolved states in the EC solution. The EC molecules were randomly placed in the unit cell, and the SFC was allocated on the graphite electrode or inside the EC solution.

As model surfaces, we used zigzag edge surfaces of graphite electrodes terminated by $\mathrm{H}$, mixed $\mathrm{H} / \mathrm{OH}$ and mixed $\mathrm{H} / \mathrm{O}$. To mimic the charging condition of the negative electrode, a Li-intercalated graphite was used. The graphite electrodes consist of four graphite sheets, where each sheet has $5 \times 6$ hexagonal rings. They are modeled as $\mathrm{C}_{288} \mathrm{H}_{46} \mathrm{Li}_{12}, \mathrm{C}_{288} \mathrm{O}_{2} \mathrm{H}_{46} \mathrm{Li}_{12}$, and $\mathrm{C}_{288} \mathrm{O}_{2} \mathrm{H}_{44} \mathrm{Li}_{12}$ for $\mathrm{H}-, \mathrm{H} / \mathrm{OH}$, and $\mathrm{H} / \mathrm{O}$-terminated graphite, respectively. The SFC aggregate is composed of 12 SFC molecules. 67, and $60 \mathrm{EC}$ molecules were stuffed into the remaining space of the supercell for the estimation of the adhesive energy of the SFC monomer and aggregate, respectively. The unit cells sizes are $14.913 \times 48.932 \times 14.824 \AA$ for monomer absorption and $14.913 \times 53.811 \times 14.824$ for aggregate adhesion.

Analysis with CBC-DFT. - In this work, we also used DFT methods with $\mathrm{CBC}$ as implemented in Gaussian $09^{53}$ to comprehensively search for the reaction pathways to possible SFCs from EC and VC molecules. This enables us to evaluate properties of single SFCs more clearly. The exchange and correlation functionals used were $\mathrm{PBE}^{45,46}$ with a $6-311++G(d, p)$ basis set and the geometries were fully optimized. In the analyses, the solvent effect was dealt with the PCM method with parameters for an EC bulk solution (dielectric constant $\varepsilon=89.78)$.

\section{Results}

SFCs in the presence of VC.- To extract the characteristic structures of VC-derived SFCs, we first examined the intermolecular reactions involving VC in detail. In our previous study with DFT-MD free energy calculations, ${ }^{38}$ we compared the initial reductive decomposition processes of $\mathrm{EC}$ and $\mathrm{VC}$ molecules in EC solvent, and elucidated a novel but more probable $\mathrm{VC}$ role in the reductive decomposition: $\mathrm{VC}$ reactivity to a reduced anion radical. We found a large exothermic reaction between the intact $\mathrm{VC}$ and $\mathrm{EC}$ anion radical, which is accompanied by the $\mathrm{CO}_{2}$ gas evolution that was experimentally observed. The product of this reaction can be regarded as one of the candidate fundamental SFC in the EC solvent with VC additive. In the previous study, we obtained intrinsic features of SFC formation in the presence of VC additives, which are summarized in Figure 2. In this scheme, a radical molecule attacks the $\mathrm{C}_{\mathrm{E}}$ or $\mathrm{C}_{\mathrm{C}}$ site of the $\mathrm{VC}$, and any radical is the candidate. 


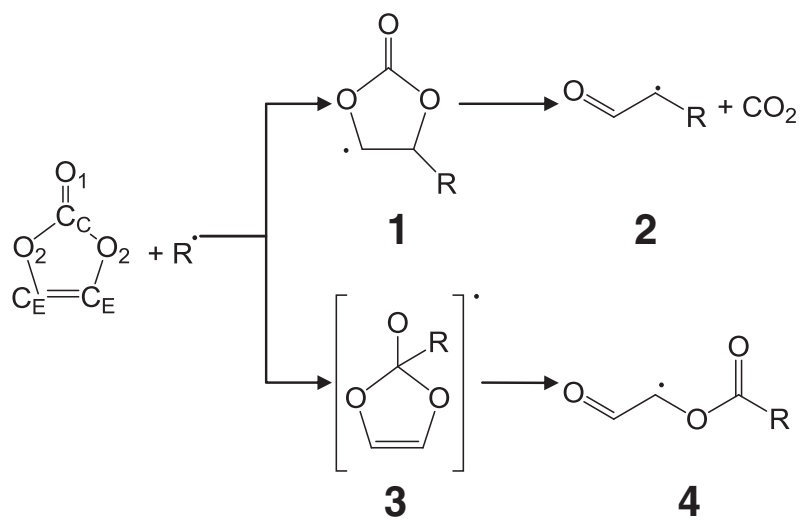

Figure 2. Reaction pathways between a radical and intact VC molecule, probable in the presence of $\mathrm{VC}$ additive.

In this study, we examined, in particular, the details of the possible reactions between an undecomposed $\mathrm{VC}$ anion radical $\left(\mathrm{VC}^{-}\right)$ and an intact VC molecule, by CBC-DFT calculations. These will be dominant in the high $\mathrm{VC}$ concentration case, because $\mathrm{VC}^{-}$anion is expected to have a larger probability of binding to another intact
VC before its decomposition. Note that we have already shown that decomposed $\mathrm{VC}$ anion radical have less reactivity against $\mathrm{VC}$ in our previous study. ${ }^{38}$ The energy diagrams of the possible $\mathrm{VC}^{-}-\mathrm{VC}$ reactions are summarized in Figure 3. All of the reactions are possible because of the large exothermic reaction energies over $20 \mathrm{kcal} / \mathrm{mol}$.

In particular, it is most plausible that $\mathrm{VC}$ reacts at the $\mathrm{C}_{\mathrm{E}}$ site of $\mathrm{VC}^{-}$for subsequent $\mathrm{CO}_{2}$ gas evolution. The most stable product of the $\mathrm{VC}-\mathrm{VC}^{-}$reaction is 7 in Figure $3 \mathrm{a}$ which is formed by release of two $\mathrm{CO}_{2}$ molecules after the reaction between the two $\mathrm{C}_{\mathrm{E}}$ sites. Indeed, experiments involving VC-only electrolyte soluition ${ }^{20}$ have reported that $\mathrm{CO}_{2}$ gas dominantly evolves even without the EC solvent. The present pathways can elucidate the probable mechanism of the experimental observation. In addition, we carried out DFT-MD simulations of the binding between $\mathrm{VC}^{-}$and $\mathrm{VC}$ through their $\mathrm{C}_{\mathrm{E}}$ atoms in the 30 $\mathrm{EC}$ solvent molecules and found that $\mathrm{CO}_{2}$ gas is spontaneously generated within $0.1 \mathrm{ps} \mathrm{MD}$ run. These results confirm that the reaction of $\mathrm{VC}$ and $\mathrm{VC}^{-}$with $\mathrm{CO}_{2}$ evolution is very plausible.

We next investigated the successive oligomerization reactions of the products denoted above, which still retained radical characters. In fact, the oligomer components have been detected in experiments. ${ }^{20}$ We examined possible reaction pathways of two successive radical reactions of $\mathrm{VC}$ species, where a ring-opened $\mathrm{EC}$ anion radical $\left(\mathrm{O}_{\mathrm{E}^{-}}\right.$ $\left.\mathrm{EC}^{-}\right)^{38}$ was used as the reaction initiator. The energy diagrams are shown in Figure 4. In the presence of VC additives, further oligomerization with VC was actually possible via very exothermic reaction.
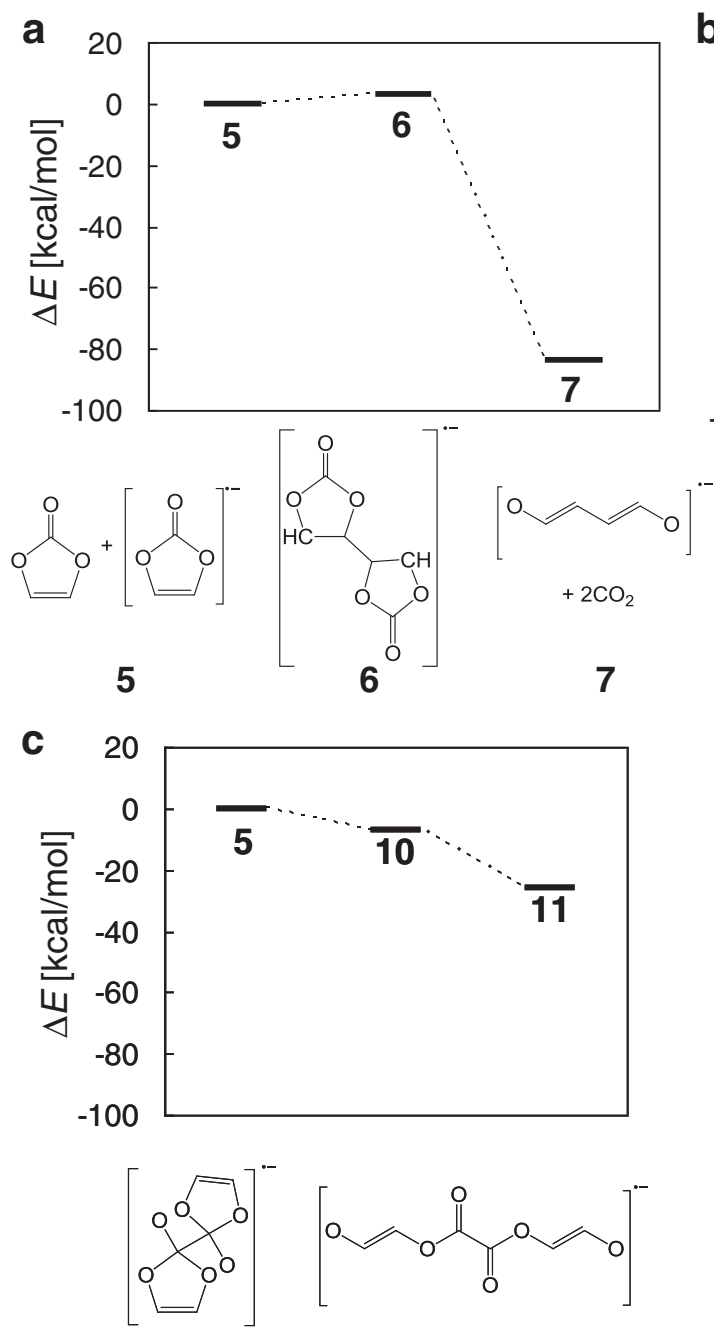

10 b

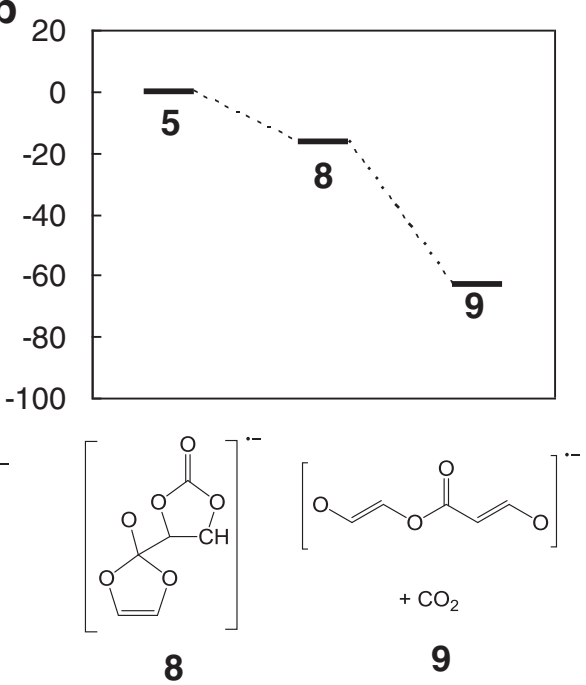



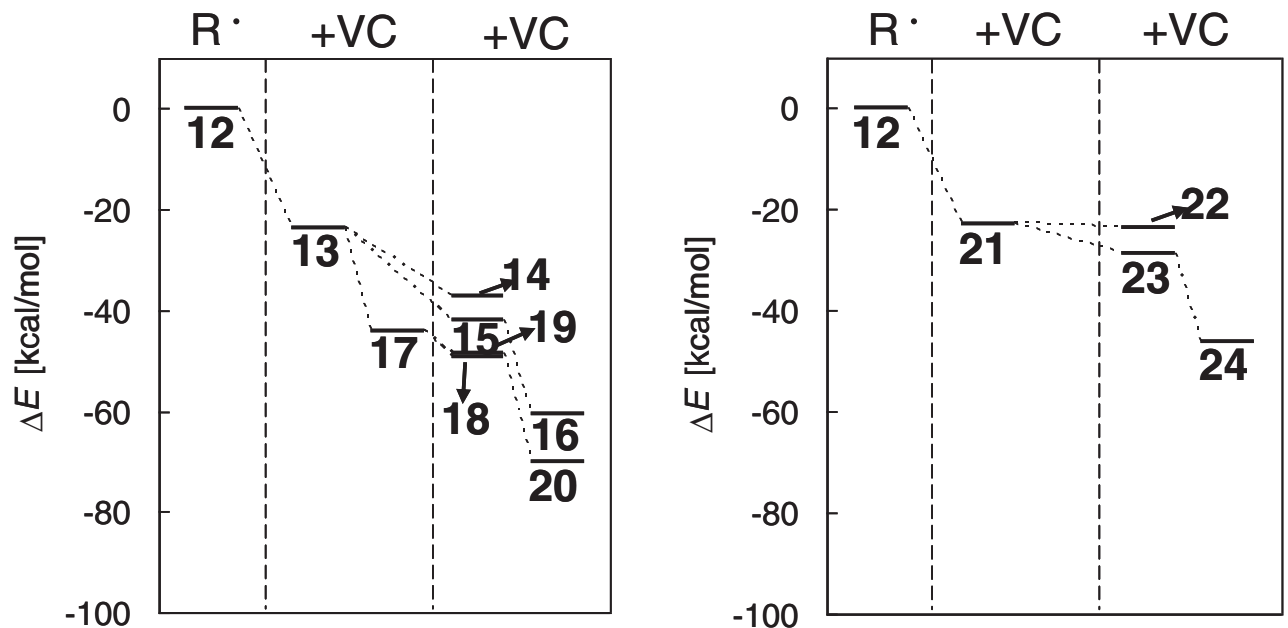

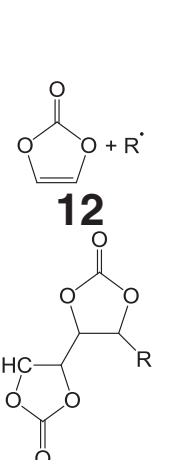

15<smiles>[R]C1OC(=O)OC1CO</smiles>

14<smiles>O=C1OC(=O)C(CCO)C1CCO</smiles>

16<smiles>O=[R6]/C=C/O</smiles>

17<smiles>[R]C(C=O)CC(=O)OC=CO</smiles><smiles>[R]C(C=O)C1COC(=O)O1</smiles>
19<smiles>[R]C(C=O)C=CO</smiles>

20<smiles>[R]OC(=O)C(C=O)OC([R20])=O</smiles><smiles>[R]C(=O)OC(C=O)C(C=O)OC([R])=O</smiles><smiles></smiles>

Figure 4. Energy diagrams of reactions between ring-opened EC anion radical (R·) and two VCs. 12 is the reactant (left side of the graph), 13, $\mathbf{1 7}$ and $\mathbf{2 1}$ are the products of R· and one VC (center of the graph), and 14-16, 18-20, and 22-24 are the products of R· and two VC (right side of the graph).

The release of $\mathrm{CO}_{2}$ during the reaction then gives more stable products as noted above. Here we emphasize that product stability via $\mathrm{CO}_{2}$ evolution is a characteristic of $\mathrm{VC}$ additives.

All of the products derived from $\mathrm{VC}$ have an $\mathrm{RC}=\mathrm{CHO}$ (vinylene alkoxide) moiety generated by cleavage of the $\mathrm{C}_{\mathrm{C}}-\mathrm{O}_{2}$ bond after the radical attack on the VC. Indeed, our previous study with DFT-MD has shown the breaking preference of the $\mathrm{C}_{\mathrm{C}}-\mathrm{O}_{2}$ bond in the reductive decomposition of $\mathrm{VC}$ for retaining $\pi$ conjugation around the double bond. ${ }^{38}$ Accordingly, we regard the $\mathrm{RC}=\mathrm{CHO}$ moiety as the main structural feature of VC-derived SFC. This feature is consistent with the experimental observation of the $\mathrm{C}=\mathrm{C}$-O moiety in the $\mathrm{VC}$-derived SEI. ${ }^{20}$ and contrasts with the well-known appearance of the $\mathrm{ROCO}_{2}$ (carbonate) terminal at the $\mathrm{C}_{\mathrm{E}}-\mathrm{O}_{2}$ bond cleavage in the reductive decomposition of EC. ${ }^{32,38}$ For the EC-derived SEI, we consider the $\mathrm{ROCO}_{2}$ moiety to be a characteristic, because it was actually observed in both experiments ${ }^{5,20}$ and calculations. ${ }^{32,38}$

On the basis of the present results and the experimental information, ${ }^{5,20}$ we prepared fundamental minimum models of SFCs for the computational modeling of SEI. Owing to the higher electron affinities (EAs) of VC and EC than those of linear carbonates commonly used, like DMC and DEC, the main organic components of SEI are likely to be composed of the reductive decomposition products of EC and VC. Note that the EAs of those molecules in EC solvents are compared in Table S.5 in the Supporting Information. Then, we constructed our model SFCs based on the EC- and VC-originated moieties. The models are summarized in Figure 5. These SFCs consist of two molecules (EC or $\mathrm{VC}$ ) and two $\mathrm{Li}$ atoms, and have either an $\mathrm{ROCO}_{2}$ or an $\mathrm{RC}=\mathrm{CHO}$ moiety for EC-derived and $\mathrm{VC}$-derived

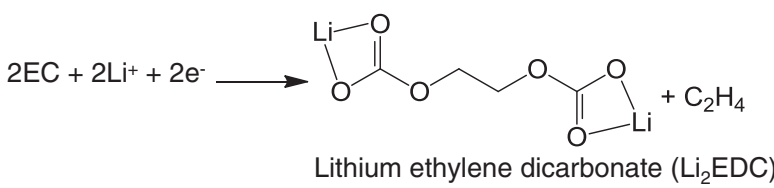

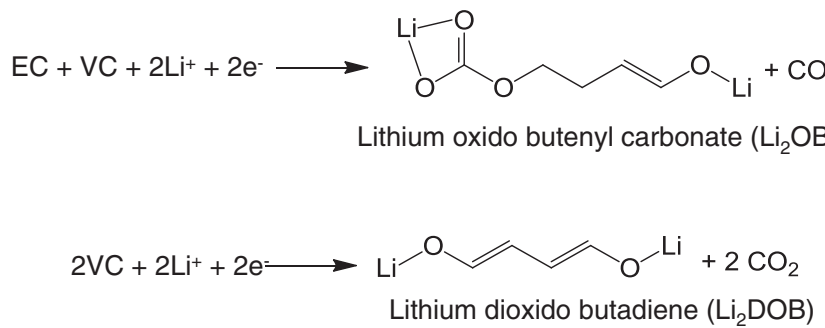

Figure 5. Fundamental reactions for the minimum models of SFCs. 
SEIs, respectively. As we have indicated, $\mathrm{Li}_{2} \mathrm{EDC}$ is the most probable model in the case of EC only. The lithium oxido butenyl carbonate $\left(\mathrm{Li}_{2} \mathrm{OBC}\right)$ and the lithium dioxide butadiene $\left(\mathrm{Li}_{2} \mathrm{DOB}\right)$ correspond to the most stable EC-VC product (17) and VC-VC product (7), respectively. These are likely to correspond to the systems under low and high VC concentrations, respectively.

SFC aggregation and adhesion. - Using the defined SFC minimum models, we investigated the structural and thermodynamic properties of SFC aggregates in the EC solvent. We prepared several initial configurations with classical MD for each SFC species $\left(\mathrm{Li}_{2} \mathrm{EDC}\right.$, $\mathrm{Li}_{2} \mathrm{OBC}, \mathrm{Li}_{2} \mathrm{DOB}$ ), and then carried out multiple DFT-MD samplings of the corresponding SFC condensed phase with PBC. Referring to the average energies, we determined probable configurations and the chemical potential of each SFC aggregate. On the other hand, we also calculated the equilibrium energy of the system involving one SFC in the EC solvent by DFT-MD. We then used Eq. 1 to evaluate the dissolution energy of each SFC species into the EC solvent.

The estimated dissolution energies of $\mathrm{Li}_{2} \mathrm{EDC}, \mathrm{Li}_{2} \mathrm{OBC}$ and $\mathrm{Li}_{2} \mathrm{DOB}$ were $+12.2,+3.1$, and $+6.1 \mathrm{kcal} / \mathrm{mol}$, respectively. This indicates that the SFC dissolution is energetically unfavorable for all SFCs examined here. The larger value for $\mathrm{Li}_{2} \mathrm{EDC}$ may imply that a grown aggregate of $\mathrm{Li}_{2} \mathrm{EDCs}$ is harder to be contracted. However, the difference between the three SFC species is very subtle, when the present statistical errors are taken into account (See Table S1 in the Supporting Information).

The present aggregation preference result differs from the results of the previous force-field calculations by Tasaki et al. ${ }^{26}$ Their calculations suggest that $\mathrm{Li}_{2} \mathrm{EDC}$ has a large exothermic heat of dissolution in the EC solvent, $-14.04 \mathrm{kcal} / \mathrm{mol}$. This can be attributed to overestimation of the solvation energy in the force-field calculations with point charges. Recently, Borodin et al. reconstructed the force-field parameters and reproduced the aggregation properties of $\mathrm{Li}_{2} \mathrm{EDC}{ }^{28}$ These indicate that careful treatment of force-field parameters could remedy the problem, but such transferability is usually questionable. Therefore, DFT treatment as in this study is crucial.

To further elucidate the aggregation process, we also used the CBC-DFT technique to calculate the binding energies between the $\mathrm{Li}_{2} \mathrm{EDC}$ and $\mathrm{Li}_{2} \mathrm{DOB}$. Supposing dimerization through the edge of the $\mathrm{LiO}_{2}$ or $\mathrm{LiO}$ moieties, we obtained 13 and $18 \mathrm{kcal} / \mathrm{mol}$, respectively, for $\mathrm{Li}_{2} \mathrm{EDC}$ and $\mathrm{Li}_{2} \mathrm{DOB}$. This result indicates that the initial aggregation can be energetically preferable. On the other hand, the different tendencies of the dissolution energy above suggest that comparison between the EC-derived and VC-derived SFCs is quite subtle, because various types of binding exist in the aggregates.

The structural features of the SFC aggregates are worth examining. The radial distribution functions (RDFs) and coordination numbers (CNs) in the probable SFC aggregates are displayed in Figure S3 in the Supporting Information and Figure 6. The results show that the $\mathrm{Li}$ ions are always surrounded by only oxygen, and the RDFs of the oxygen atoms from $\mathrm{Li}$ ions show large first peaks around $1.9 \AA$ in all the SFC aggregates. This implies that the "glue roles" of the $\mathrm{Li}$ ions are not so different among these aggregates. The averaged CNs in the first shell of the $\mathrm{Li}$ ion (up to $2.5 \AA$ ) are 4.4, 3.7, and 2.9 for $\mathrm{Li}_{2} \mathrm{EDC}$, $\mathrm{Li}_{2} \mathrm{OBC}$, and $\mathrm{Li}_{2} \mathrm{DOB}$, respectively. Hence the CNs largely depend on the ratio of the number of $\mathrm{O}$ atoms and $\mathrm{Li}$ ions in system. It is also found that the edge $\mathrm{O}$ atoms in the $\mathrm{ROCO}_{2}$ moiety have a $\mathrm{CN}$ of about 2 for $\mathrm{Li}$ coordination, whereas $\mathrm{CN}=3$ is roughly assigned to the $\mathrm{RO}$ moiety. This means that two and three SFCs participate in one joint through the $\mathrm{Li}$ glue for $\mathrm{Li}_{2} \mathrm{EDC}$ and $\mathrm{Li}_{2} \mathrm{DOB}$, respectively.

Typical configurations in the SFC aggregates are shown in Figure 7. In the stable $\mathrm{Li}_{2} \mathrm{EDC}$ aggregate, two $\mathrm{ROCO}_{2}$ edges mainly contribute to the so called four-fold coordination to the $\mathrm{Li}$ ion, suggesting that the networking with $\mathrm{Li}$ ion bridging is a typical feature. However, the ether-type oxygen (-COC-) may also join the coordination, leading to the $\mathrm{CN}$ over 4 . We also point out that the $\mathrm{Li}_{2} \mathrm{EDC}$ can easily have bent form, contributing to the $\mathrm{CN}$ increase as well. These structural features are consistent with the previous studies by

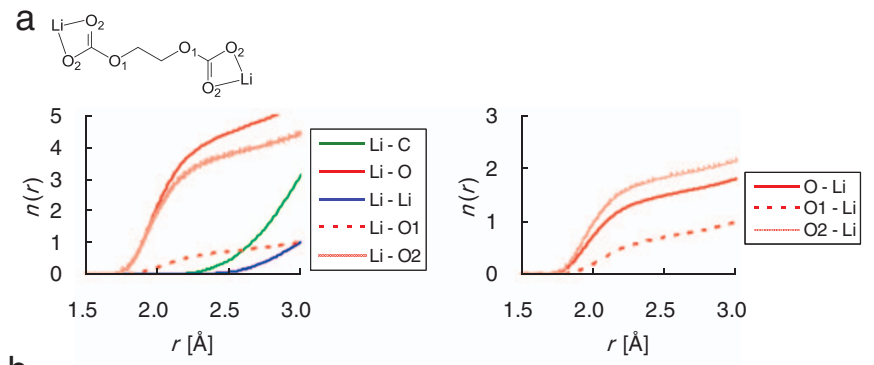

b
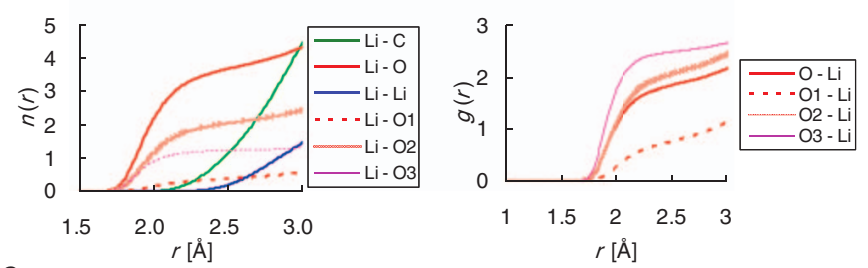

$\mathrm{C}$
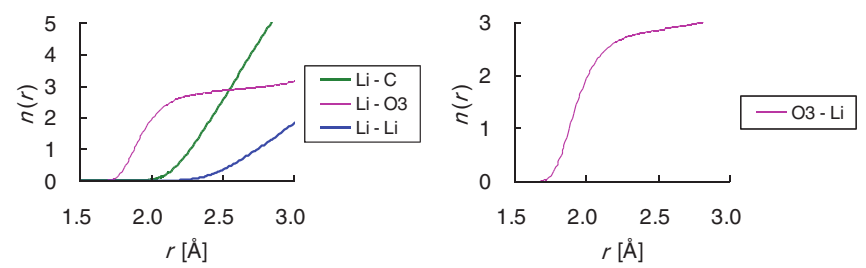

Figure 6. Coordination numbers (CNs) from $\mathrm{Li}$ ions (left side) and oxygen atoms (right side) in the SFC aggregates consisting of (a) $\mathrm{Li}_{2} \mathrm{EDC}$, (b) $\mathrm{Li}_{2} \mathrm{OBC}$, and (c) $\mathrm{Li}_{2}$ DOB. Different types of oxygen atoms such as $\mathrm{ROCO}_{2}$ and $\mathrm{RO}$ are discriminated.

ab initio CBC calculation, ${ }^{30,31}$ classical MD calculation ${ }^{28}$ and DFTMD calculation. ${ }^{36}$ On the other hand, rather straight SFCs with $\pi$ conjugation in the $\mathrm{Li}_{2} \mathrm{DOB}$ provide smaller CNs. This may affect the aggregation preference as well. In summary, the SFC connections are mainly formed with the edge moieties of $\mathrm{ROCO}_{2}$ and $\mathrm{RO}$, and the bridging behaviors via the Li glues significantly depend on the SFC characteristics.

In order to understand the SEI formation and the mechanism of surface growth, the adhesive property of the SFCs on the negative electrode is also indispensable. Here we calculated the average adhesive energies of single SFCs and SFC aggregates to the hydrogen-terminate graphite edge, a typical model of a negative electrode under reductive condition, by DFT-MD sampling. In this analysis, we focused on $\mathrm{Li}_{2} \mathrm{EDC}$ and $\mathrm{Li}_{2} \mathrm{DOB}$ to clearly extract the intrinsic characteristics of EC- and VC-derived SFCs.

We first described the adhesion of the single SFCs. For the adhesive state, we prepare an initial configuration where the SFC monomer has a "stand-up" structure where the molecular axis was perpendicular to a

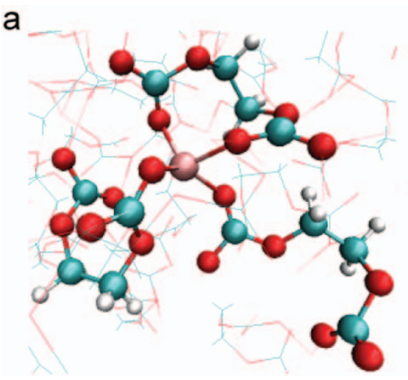

b

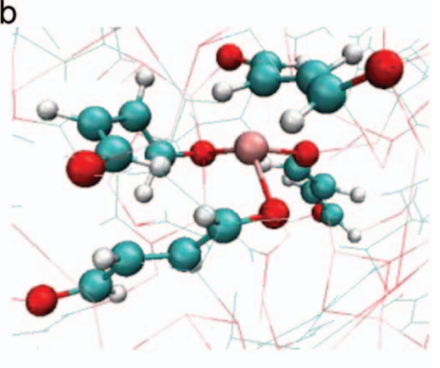

Figure 7. DFT-MD snapshots of the typical configurations in the SFC aggregates. (a) $\mathrm{Li}_{2} \mathrm{EDC}$ (b) $\mathrm{Li}_{2} \mathrm{DOB}$. 


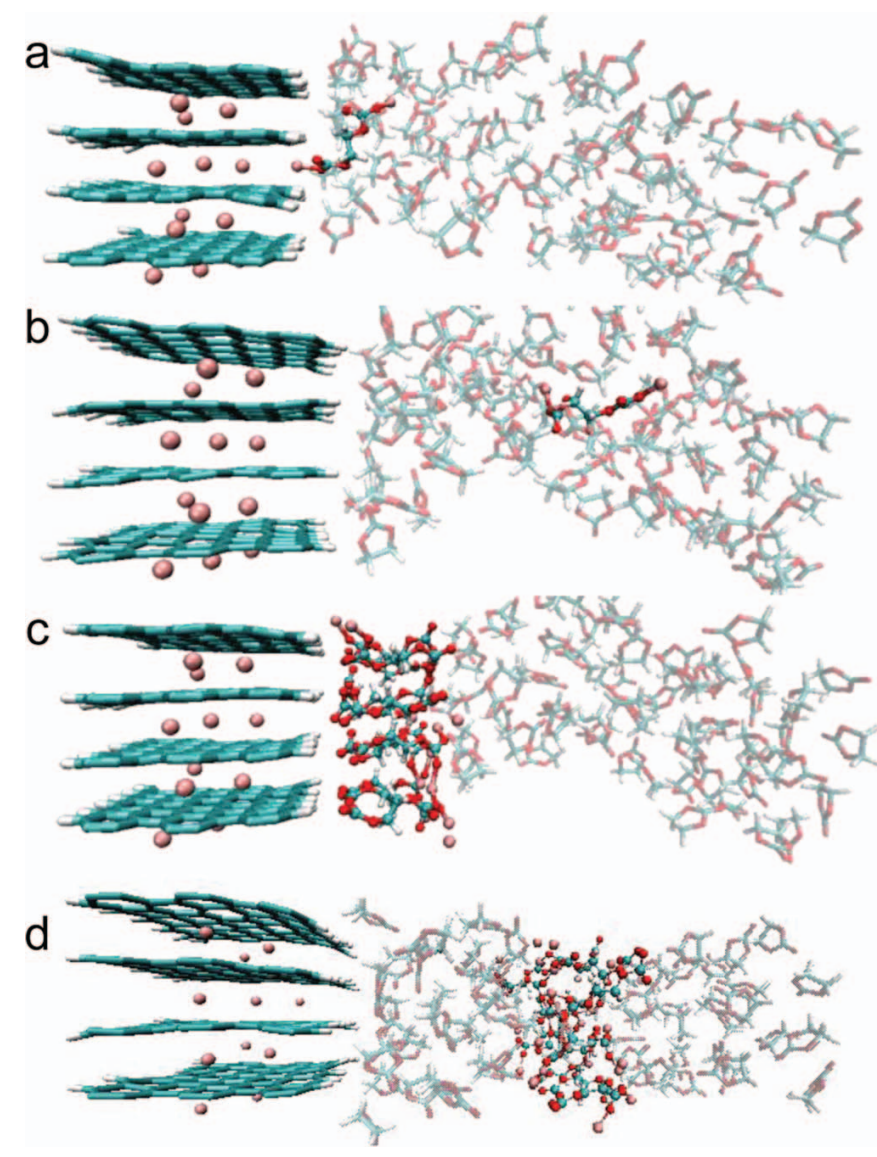

Figure 8. DFT-MD Snapshots of electrode/electrolyte interphase with $\mathrm{Li}_{2} \mathrm{EDC}$. Graphite and intercalated $\mathrm{Li}$ are shown as tube style (left side). $\mathrm{Li}_{2} \mathrm{EDC}$ is shown as ball and stick style (center). EC solvent molecules are shown by diaphanous tube style (right side). Cyan, white, red and pink colors denote $\mathrm{C}, \mathrm{H}, \mathrm{O}$, and $\mathrm{Li}$ atoms, respectively. (a) Adhesion structure of one $\mathrm{Li}_{2} \mathrm{EDC}$ monomer on the graphite electrode. (b) Dissolution structure of single $\mathrm{Li}_{2} \mathrm{EDC}$ molecule in EC solvent. (c) Adhesion structure of $12 \mathrm{Li}_{2} \mathrm{EDC}$ aggregate on graphite electrode. (d) Dissolution structure of $12 \mathrm{Li}_{2} \mathrm{EDC}$ aggregate in EC solvent.

the surface and a Li ion was put between the SFC and the graphite edge. On the other hand, the SFC monomer was immersed in the EC solvent for the dissolution state. Snapshots of the equilibrium trajectories for the $\mathrm{Li}_{2} \mathrm{EDC}$ are displayed in Figures 8a and 8b. In the attached state, it is apparent that the surface $\mathrm{Li}$ ion is located at a site bridging the two graphite sheets. This is attributed to the repulsive interaction between the terminal $\mathrm{H}$ and the $\mathrm{Li}$ ion. A similar scenario appears in the $\mathrm{Li}_{2} \mathrm{DOB}$ case as well.

The average adhesive energies of the single SFCs are listed in Table I. We found that adhesion of the monomer on the graphite surface in the EC solvent is energetically unfavorable for both $\mathrm{Li}_{2} \mathrm{EDC}$ and $\mathrm{Li}_{2} \mathrm{DOB}$. Although the former adhesion appeared less probable, we concluded that there was no particular difference between the EC- and VC-derived SFCs. The results seem inconsistent with the surface growth mechanism, which intrinsically assumes successive SFC adhesions.

Table I. Adhesive energies (in kcal/mol) of SFC monomers and aggregates per component on $\mathrm{H}$-terminated graphite edge surface.

\begin{tabular}{ccc} 
& Single SFC & SFC aggregate \\
\hline $\mathrm{Li}_{2}$ EDC & +10.6 & +3.2 \\
$\mathrm{Li}_{2}$ DOB & +4.2 & +5.5
\end{tabular}

To check for possible calculation errors, we carried out some complementary calculations. Considering the energy dependence on the structural difference, we examined the $\mathrm{Li}_{2} \mathrm{EDC}$ monomer with the "liedown" structure, in which the SFC lies parallel to the graphite surface. The calculated adhesive energy was still positive, $+7.0 \mathrm{kcal} / \mathrm{mol}$. We also checked for the effect of vdW interaction by using the DFT-D technique. The estimated adhesive energy for "stand-up" structure of $\mathrm{Li}_{2} \mathrm{EDC}$ was about $+4.9 \mathrm{kcal} / \mathrm{mol}$. Finally, we examined the effect of graphite edge termination. The average adhesive energies to the $\mathrm{H} / \mathrm{OH}-$ and $\mathrm{H} / \mathrm{O}$ - terminated graphite electrode were found to be about +1.0 and $+3.4 \mathrm{kcal} / \mathrm{mol}$. Despite the expectation that the $\mathrm{vdW}$ interaction and $\mathrm{OH}$ or $\mathrm{O}$ terminations might change the sign of adhesive energy, the above results still showed positive values. We therefore conclude that the single SFCs do not have a significant adhesion preference on the standard graphite electrode in the EC solution.

In addition to single SFC adsorption, the adhesion preference of the SFC aggregates is also important. Using the stable SFC aggregate structures obtained in this work, we carried out DFT-MD samplings of the adhesion and dissolution states. Snapshots of the equilibrium trajectories for the $\mathrm{Li}_{2} \mathrm{EDC}$ aggregate are displayed in Figures $8 \mathrm{c}$ and $8 \mathrm{~d}$. The estimated adhesive energies listed in Table I indicate that the SFC aggregates also prefer the dissolved state in the EC solution. Although the estimated statistical errors of several $\mathrm{kcal} / \mathrm{mol}$, as listed in Table S3, make the conclusion slightly difficult, all the six trajectories clearly show that the SFC monomer adhesion is energetically unfavorable. Furthermore, the whole adhesive energies of the SFC aggregates, before being divided by the number of SFC constituents, are several tens of $\mathrm{kcal} / \mathrm{mol}$ and large enough compared with the statistical errors. Therefore, we concluded that the SFCs do not have a significant adhesion preference to the graphite edge surface in the EC solution. We also found that there was little difference between EC- and VC-derived SFCs as was in the case of the monomer. These results indicate that growth of the SFC aggregate generally relies on the intrinsic aggregation preference in the EC solvent rather than on the adhesions of SFCs to the graphite edge surfaces.

Electronic states of SFC aggregates. - Calculated quantities regarding the SFC aggregation and adhesion imply that the surface growth mechanism is questionable. To clarify this point, we examined the electronic insulation of the probable SFC aggregate in the EC solution. It is experimentally reported that thickness of VC-derived SEI films is thinner than that of EC-derived SEI, which is typically several tenths of nanometers. ${ }^{8,9}$ The surface growth mechanism suggests that better electronic insulating of the VC-derived SFC aggregates suppresses electron tunneling from the electrode to the electrolyte solution and decreases electrolyte reductive decompositions, the result being a thinner SEI film. The electronic insulation of possible SEI components is thus an important key for SEI formation.

We calculated the PDOSs of snapshots of the equilibrium trajectories obtained by the DFT-MD simulations of the systems involving graphite surface, SFC aggregate and EC solution (Figure 9). It is noteworthy that we examined $\mathrm{Li}_{2} \mathrm{EDC}$ and $\mathrm{Li}_{2} \mathrm{DOB}$ cases to extract the difference between the EC- and VC-derived SFCs. We found that both SFCs have higher unoccupied energy level more than $1 \mathrm{eV}$ higher than is the case for the EC solvent. Even in the EC-only systems, $\mathrm{Li}_{2} \mathrm{EDC}$ is quite insulating against the EC solvent. More importantly, VC additives do not significantly improve the electronic insulating property.

These results were also supported by the CBC-DFT calculations of the electron affinities of $\mathrm{Li}_{2} \mathrm{EDC}$ and $\mathrm{Li}_{2} \mathrm{DOB}$ dimers relative to the EC solvent. The orbital that accepts the injected electrons is the $\mathrm{Li} 2 \mathrm{~s}$ orbital for both SFCs and its energy level is higher than that of the $\pi^{*}$ orbital around the $\mathrm{CO}_{3}$ moiety for the $\mathrm{EC}$ solvent. These results again indicate that electronic insulations of the SFCs are not so different and are high enough to prevent EC reduction. Therefore, VC-derived SFCs do not enhance the insulating property in general. Detailed results of the CBC-DFT calculations are summarized in Supporting Information Table S4.

In summary, the present results demonstrate that the $\mathrm{VC}$ additive does not significantly improve the adhesion to the graphite surface 

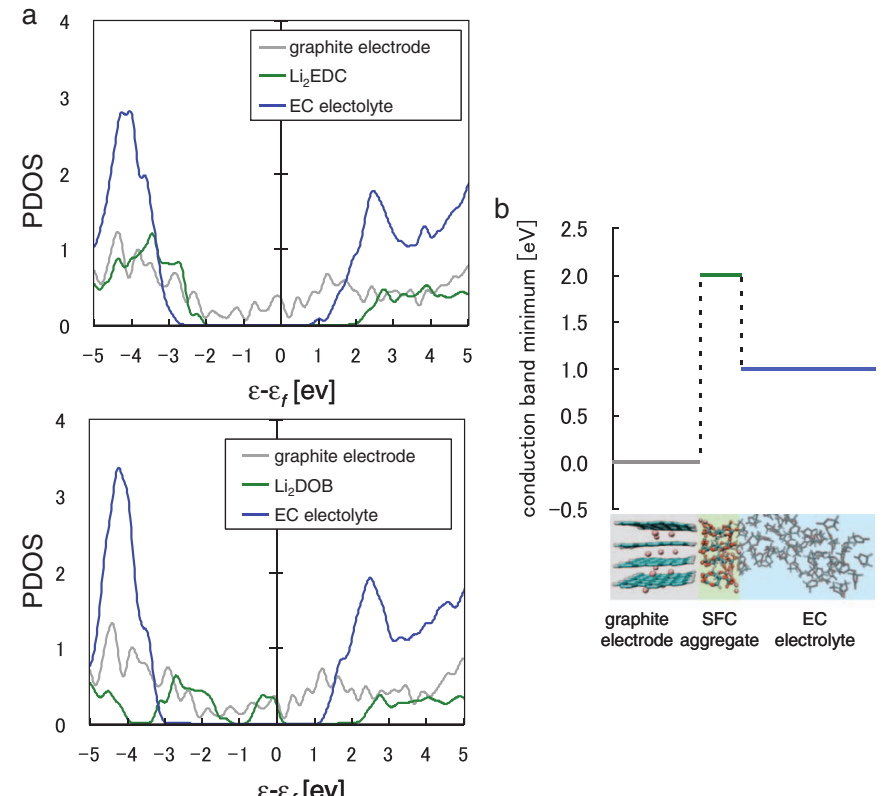

Figure 9. (a) Projected densities of states (PDOSs) of snapshots taken from the DFT-MD simulations. $\mathrm{Li}_{2} \mathrm{EDC}$ aggregate on graphite in EC solution (up), and $\mathrm{Li}_{2} \mathrm{DOB}$ aggregate on graphite in $\mathrm{EC}$ solution (down). The energy origin is set to Fermi energy. (b) Schematic conduction band minimum levels in the system.

and insulating properties. Therefore, the additive effects, such as the decrease of the irreversible capacity, do not rely on the properties of the resultant SEI film stability on the electrode surface, but instead depend on its reaction mechanism, as our previous study pointed out. ${ }^{38}$

\section{Discussion}

In this section, we discuss the probable mechanism of SEI film formation based on the results of our calculations. First, we consider the "surface growth mechanism", which has been a simple view of SEI formation based on precipitation of reductive decomposition products of the electrolyte molecules (e.g. Fig. 2 in Ref. 23). The "surface growth mechanism" involves precipitations of the SFCs formed by reductive decompositions of the electrolyte on the negative electrode interface, and the SFC aggregation progresses from the surface nucleation. The growth then stops when the SEI film is thick enough to prevent further electrolyte reduction (Figure 10a). However, the present results demonstrate that the adhesion of SFC aggregate on the electrode is less stable in the EC solution, and the EC-derived SFC aggregates have almost the same electronic insulating properties as the VC-derived aggregates. These results clearly indicate that "surface growth mechanism" does not play a major role in SEI film formation.

We then propose a new mechanism of SEI film formation based on the present results, which we named the "near-shore aggregation mechanism", a schematic scheme of which is shown in Figure 10b. In this mechanism, the electrolyte reductive decompositions always take place on the negative electrode surface, and the formed SFCs desorb into the electrolyte solution. Aggregation of the SFCs then proceeds in the "near-shore region" from the electrode, which is a crucial difference from the "surface growth" scenario. These processes are well explained by the calculated tendencies of aggregation and adhesion. When the SFC clusters grow to a certain size in the near-shore region as well as in the electrolyte solution and start to coalesce, the free electrolyte region between the electrode and the SFC aggregates shrinks due to further supply of SFCs on the electrode surface. This scenario can explain why the SEI thickness can reach on the order of $10 \mathrm{~nm}$ order despite the fact that the SFC aggregates have high electronic insulation. Note that continuous supply of the electrolyte molecules on the electrode surface in the present mechanism is also crucial for the thickness. Once the SFC clusters (or films) cover the electrode surface, the SFC supply from the electrode slows down and the film adhesion can be kept in a metastable state because the large size of the aggregate will prevent the corrective desorption of the film. This is a scenario deduced from our calculated results.

This "near-shore aggregation" mechanism may also give several probable suggestions. Regarding the VC-additive effect (reduction of SEI thickness ${ }^{8,9}$ and low irreversible capacity loss. ${ }^{7}$ ), more rapid contact between the SFC aggregate and the electrode surface is likely to play an essential role in reducing the thickness of the VC-derived SEI films. This factor can be explained by the oligomerization tendency of VC. As we have shown in Results section, intact VC will easily oligomerize with reduction products during the SFC formation, which is actually supported by the experimental observation of the oligomer products of VC molecules. ${ }^{20}$ This accelerates the intermolecular O-Li-O coordination and thus growth of the SFC aggregate, which gradually facilitate the contact of the SFC aggregate to the electrode surface. The thinner thickness eventually causes less irreversible capacity.

In addition, the near-shore mechanism suggests a possibility of exfoliation of the SEI film and the following regeneration during the charge-discharge cycles, ${ }^{3,4}$ which is analogous to SFC desorption and contact of the SFC clusters with the electrode. However, there will be several alternative mechanisms for the subsequent cycles, which will be in our future study. Note that a similar growth mechanism is proposed for $\mathrm{Li}_{2} \mathrm{O}_{2}$ in $\mathrm{Li}$-air battery. ${ }^{51}$ This may imply that the "near-shore aggregation" concept can be universal.

Here, we discuss the limitations of the present modeling for the SEI formation. First of all, this work does not involve the inorganic

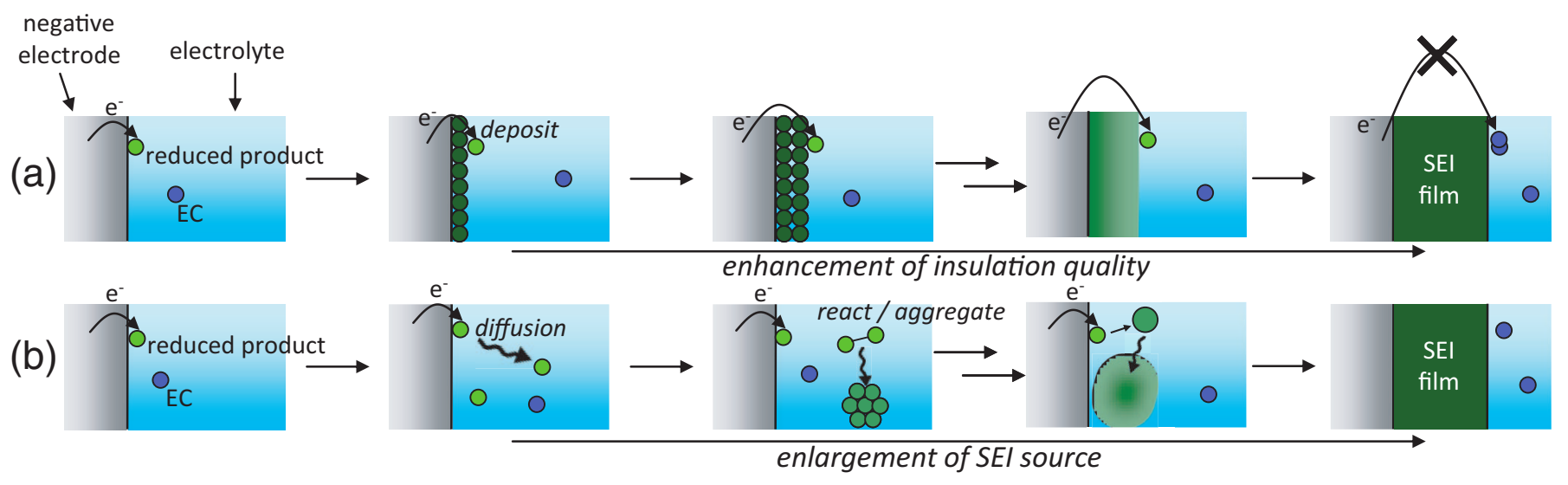

Figure 10. Schematic pictures of SEI film formation processes based on (a) surface growth mechanism, and (b) near-shore aggregation mechanism. 
products of the reductive decomposition. The inorganic components, i.e., $\mathrm{LiF}, \mathrm{Li}_{2} \mathrm{O}, \mathrm{Li}_{2} \mathrm{CO}_{3}$, also consist of the SEI film. There are reports suggesting that the inorganic components exist near the graphite electrode, followed by an organic or polymeric layer close to the electrolyte phase. ${ }^{5,17-19}$ In fact, recent X-ray photoelectron spectroscopy (XPS) measurements surely indicate that $\mathrm{LiF}$ and $\mathrm{Li}_{2} \mathrm{CO}_{3}$ plenty exist on the graphite electrode surface. ${ }^{22}$ On the other hand, it is also reported that distinguishing between graphite and $\mathrm{CC} / \mathrm{CH}$ bonds is subtle in the XPS and the XPS imaging indicates many microscopic pores for the organic components between the inorganic species (e.g. Fig. 8 in Ref. 22). Furthermore, it is regarded that the organic SFCs have faster $\mathrm{Li}$-ion transport and thus more responsibility for that than the inorganic ones. Therefore, the present modeling that takes into account the interface between the organic SFCs and the reduced graphite electrode surface is still relevant. In fact, the typical SEI film thickness cannot be explained by the full cover of the electrode surface by the inorganic components having large band gaps in general, which should have prevented the reduction of the electrolyte molecules quickly. ${ }^{40}$ Of course, the effect of the inorganic components on the aggregation and adhesion of the organic SFCs is essential for full understanding of the SEI formation mechanism. An investigation of such issue is underway in our next study. As an example, the preliminary result on adhesion of $\mathrm{LiF}$ aggregates to the $\mathrm{H}$-capped graphite surface in $\mathrm{EC}$ electrolyte was shown in Figure S6 in the Supporting Information. This implies that the inorganic aggregates have tendency of unstable adhesion like the organic ones, which maybe related to the "near-shore aggregation" mechanism as well.

We also discuss effects of the electrode surface defects. We employed the perfect zigzag graphite with $\mathrm{H}, \mathrm{OH}$, and $\mathrm{O}$ capped structures only, and neglected the surface imperfection such as defects. On the other hand, at solid-liquid interfaces, such surface defects are easily passivated by adsorptions of molecules in the electrolyte. The passivated sites do not seem to change the adhesion tendency of the organic SFCs observed in this work very much. Even if they can be nucleation sites, it still holds that the majority of the SFCs generated at the electrode interface can diffuse to the other region (maybe near the nucleation sites) to make the aggregate. In this respect, the concept of the "near-shore aggregation" mechanism seems still relevant, although this issue is also to be clarified in the future study.

As discussed above, the present modeling involves several assumptions and limitations for the realistic electrolyte-electrode interfaces. However, we have carried out careful evaluations of the validity. Besides the DFT-MD simulations give plenty of novel aspects based on accurate description of the electronic states and the atomic interactions. We therefore believe that this work is a substantial first step to understand the SEI formation at the atomistic level and the results reasonably involve a major essence of the initial stage of SEI formation.

\section{Summary}

In this study, we used DFT calculations to examine the ability of the "surface growth mechanism" to explain the formation of the SEI at the interface between the carbonate electrolyte solution and the negative electrode. We chose most typical systems under the charging condition, involving EC solvents, VC additives and graphite edge surfaces with various types of termination. We first extracted stable SFCs for the EC/VC electrolyte and constructed probable SFC aggregates via DFT-MD. We then examined their solubility in the EC solution, the adhesion to the model graphite electrodes, and the electronic properties.

The results indicate that the adhesions of the SFC aggregates are energetically less favorable, and all the examined SFC aggregates have high electronic insulation against the EC solution. These results indicate that the "surface growth mechanism" does not play a major role in SEI film formation. Based on the present results, we instead propose a new mechanism, "near-shore aggregation mechanism," in which the SFCs formed at the electrode surface desorb to the near-shore region and form aggregates. The grown SFC aggregates coalesce and attach to the electrode to complete the SEI formation. The mechanism can naturally account for the SEI growth up to a typical thickness of several tens of nanometers, by allowing a continuous supply of electrolyte molecules to the electrode surface. The VC additive effects with thinner SEI film thickness can be also explained without relying on electronic insulation. Although there are still limitations in the present modeling, careful evaluation indicates that the present scenario certainly gives a novel perspective on the mechanism of SEI film formation. Further investigations with few limitations, necessary for the comprehensive understanding, will be addressed in future works.

\section{Acknowledgment}

K.U and Y. O. acknowledge Dr. H. Watanabe and Dr. K. Furuya of FUJIFILM Corp. for their support. This work was supported by KAKENHI 23340089 as well as by the Strategic Programs for Innovative Research (SPIRE), MEXT and the Computational Materials Science Initiative (CMSI), Japan. The calculations in this work were carried out at the super-computer centers of NIMS and the University of Tokyo, as well as on the K computer at the RIKEN AICS through the HPCI System Research Projects (Proposal Numbers hp140071 and hp140232).

\section{References}

1. W. Kohs, H. J. Santner, F. Hofer, H. Schrottner, J. Doninger, I. Barsukov, H. Buqa J. H. Albering, K.-C. Moller, J. O. Besenhard, and M. Winter, J. Power Sources., 119, 528 (2003).

2. R. Fong, U. von Sacken, and J. R. Dahn, J. Electrochem. Soc., 137, 2009 (1990).

3. T. R. Job, K. Xu, O. Borodin, and M. Ue, Electrolytes for Lithium and Lithium-Ion Batteries (Modern Aspects of Electrochemistry), Springer, Berlin, (2014).

4. Y. Wang and P. B. Balbuena, Lithium-Ion Batteries: Solid-Electrolyte Interphase, Imperial College, London, (2004).

5. P. Verma, P. Maire, and P. Novák, Electrochimica Acta, 55, 6332 (2010).

6. M. Nie, D. Chalasani, D. P. Abraham, Y. Chen, A. Bose, and B. L. Lucht, J. Phys. Chem. C., 117, 1257 (2013).

7. D. Aurbach, K. Gamolsky, B. Markovsky, Y. Gofer, M. Schmidt, and U. Heider, Electrochimca Acta, 47, 1423 (2002).

8. S.-K. Jeong, M. Inaba, R. Mogi, Y. Iriyama, T. Abe, and Z. Ogumi, Langmuir, 17, 8281 (2001).

9. O. Matsuoka, A. Hiwara, T. Omi, M. Toriida, T. Hayashi, C. Tanaka, Y. Saito, T. Ishida, H. Tan, S. Ono, and S. Yamamoto, J. Power Sources, 108, 128 (2002).

10. S.-K. Jeong, M. Inaba, T. Abe, and Z. Ogumi, J. Electrochem. Soc., 148, A989 (2001)

11. D. Aurbach, E. Zinigrad, Y. Cohen, and H. Teller, Solid State Ionics, 148, 405 (2002)

12. J. Vetter, P. Novák, M. R. Wagner, C. Veit, K.-C. Möller, J. O. Besenhard, M. Winter, M. Wohlfahrt-Mehrens, C. Vogler, and A. Hammouche, J. Power Sources, 147, 269 (2005).

13. S. S. Zhang, J. Power Sources, 162, 1379 (2006).

14. S. Leroy, F. Blanchard, R. Dedryvère, H. Martinez, B. Carré, D. Lemordant, and D. Gonbeau, Surf. Interface Anal., 37, 773 (2005).

15. J. C. Burns, R. Petibon, K. J. Nelson, N. N. Sinha, A. Kassam, B. M. Way, and J. R. Dahn, J. Electrochem. Soc., 160, A1668 (2013).

16. V. A. Agubra and J. W. Fergus, J. Power Sources, 268, 152 (2014).

17. E. Peled, D. Golodnitsky, and G. Ardel, J. Electrochem. Soc., 144, L208 (1997)

18. A. M. Andersson, A. Henningson, H. Siegbahn, U. Jansson, and K. Edström, J. Power Sources, 119-121, 522 (2003).

19. K. Edström, M. Herstedt, and D. P. Abraham, J. Power Sources, 153, 380 (2006).

20. H. Ota, Y. Sakata, A. Inoue, and S. Yamaguchi, J. Electrochem. Soc., 10, A1659 (2004).

21. B. Marom, O. Haik, D. Aurbach, and I. C. Halalay, J. Electrochem. Soc., 157(8) A972 (2010).

22. P. Niehoff, S. Passerini, and M. Winter, Langmuir, 29, 5806 (2013).

23. J. Yan, J. Zhang, Y. C. Su, X. G. Zhang, and B. J. Xia, Electrochimica Acta, 55, 1785 (2010).

24. K. Tasaki, J. Phys. Chem. B, 109, 2920 (2005).

25. K. Tasaki, A. Goldberg, J.-J. Lian, M. Walker, A. Timmons, and S. J. Harris, J. Electrochem. Soc., 156, A1019 (2009).

26. K. Tasaki and J. H. Stephen, J. Phys. Chem. C, 114, 8076 (2010).

27. O. Borodin, G. D. Smith, and P. Fan, J. Phys. Chem. B, 110, 22773 (2006)

28. O. Borodin, G. V. Zhuang, P. N. Ross, and K. Xu, J. Phys. Chem. B, 117, 7433 (2013).

29. N. Takenaka, Y. Suzuki, H. Sakai, and M. Nagaoka, J. Phys. Chem. C, 118, 10874 (2014).

30. S. Matsuoka, T. Asada, and K. Kitaura, J. Electrochem. Soc., 147, 1695 (2000).

31. Y. Wang and P. B. Balbuena, J. Phys. Chem. A, 106, 9582 (2002).

32. Y. Wang, S. Nakamura, M. Ue, and P. B. Balbuena, J. Am. Chem. Soc., 123, 11708 (2001). 
33. Y. Wang, S. Nakamura, K. Tasaki, and P. B. Balbuena, J. Am. Chem. Soc., 124, 4408 (2002).

34. K. Leung and J. L. Budzien, Phys. Chem. Chem. Phys., 12, 6583 (2010).

35. K. Leung, Phys. Chem. Chem. Phys., 17, 1637 (2015)

36. S. Ogata, N. Ohba, and T. Kouno, J. Phys. Chem. C, 117, 17960 (2013).

37. P. Ganesh, P. R. C. Kent, and D.-e Jiang, J. Phys. Chem. C, 116, 24476 (2012).

38. K. Ushirogata, K. Sodeyama, Y. Okuno, and Y. Tateyama, J. Am. Chem. Soc., 135 11967 (2013).

39. J. M. Martinez de la Hoz and P. B. Balbuena, Phys. Chem. Chem. Phys., 16, 17091 (2014).

40. L. Benitez, D. Cristancho, J. M. Seminario, J. M. Martinez de la Hoz, and P. B. Balbuena, Electrochimica Acta, 140(10), 250 (2014).

41. R. Car and M. Parrinello, Phys. Rev. Lett., 55, 2471 (1985).

42. CPMD, http://www.cpmd.org/, Copyright IBM Corp 1990-2008, Copyright MPI für Festkörperforschung Stuttgart 1997-2001.

43. S. Nose, J. Chem. Phys., 81, 511 (1984).

44. W. G. Hoover, Phys. Rev. A, 31, 1695 (1985).

45. J. P. Perdew, K. Burke, and M. Ernzerhof, Phys. Rev. Lett., 77, 3865 (1996).

46. J. P. Perdew, K. Burke, and M. Ernzerhof, Phys. Rev. Lett., 78, 1396 (1997).

47. S. Goedecker, M. Teter, and J. Hutter, Phys. Rev. B, 54, 1703 (1996).

48. C. Hartwigsen, S. Goedecker, and J. Hutter, Phys. Rev. B, 58, 3641 (1998).
49. M. Krack, Theor. Chem. Acc., 114, 145 (2005).

50. S. Grimme, J. Comp. Chem, 27, 1787 (2006).

51. A. Marquez, Mater. Chem. Phys., 104, 199 (2007).

52. Y.-K. Han, S. U. Lee, J.-H. Ok, J.-J. Cho, and H.-J. Kim, Chem. Phys. Lett, 360, 359 (2002).

53. Gaussian 09, Revision D.01, M. J. Frisch, G. W. Trucks, H. B. Schlegel, G. E. Scuseria, M. A. Robb, J. R. Cheeseman, G. Scalmani, V. Barone, B. Mennucci, G. A. Petersson, H. Nakatsuji, M. Caricato, X. Li, H. P. Hratchian, A. F. Izmaylov, J. Bloino, G. Zheng, J. L. Sonnenberg, M. Hada, M. Ehara, K. Toyota, R. Fukuda, J. Hasegawa, M. Ishida, T. Nakajima, Y. Honda, O. Kitao, H. Nakai, T. Vreven, J. A. Montgomery Jr., J. E. Peralta, F. Ogliaro, M. Bearpark, J. J. Heyd, E. Brothers, K. N. Kudin, V. N. Staroverov, R. Kobayashi, J. Normand, K. Raghavachari, A. Rendell, J. C. Burant, S. S. Iyengar, J. Tomasi, M. Cossi, N. Rega, J. M. Millam, M. Klene, J. E. Knox, J. B. Cross, V. Bakken, C. Adamo, J. Jaramillo, R. Gomperts, R. E. Stratmann, O. Yazyev, A. J. Austin, R. Cammi, C. Pomelli, J. W. Ochterski, R. L. Martin, K. Morokuma, V. G. Zakrzewski, G. A. Voth, P. Salvador, J. J. Dannenberg, S. Dapprich, A. D. Daniels, Ö. Farkas, J. B. Foresman, J. V. Ortiz, J. Cioslowski, and D. J. Fox, Gaussian, Inc., Wallingford CT (2009).

54. B. D. Adams, C. Radtke, R. Black, M. L. Trudeau, K. Zanhib, and L. F. Nazar, Energy Envirion. Sci., 6, 1772 (2013). 